\title{
Rapid access rather than open access leads to improved effectiveness of an ENT emergency clinic
}

\author{
Catherine Smyth, Michael Moran, Catherine Diver, Susanne Hampton
}

\begin{abstract}
An Ear, Nose and Throat (ENT) emergency clinic provides important access to specialist care for patients referred by General Practitioners (GPs), Emergency Departments and doctors on non-ENT hospital wards. The aim is to enable the prompt diagnosis and management of acute conditions, within an optimum environment containing appropriate clinical and staff resources.
\end{abstract}

Amid concerns that an open access ENT casualty service had become overburdened we performed a four week audit of all attendances. We identified $45 \%$ of patients presenting to the clinic without having evidence of first accessing primary care assessment or treatment. Waiting times were unpredictable, averaging 75 minutes and clinic numbers above those recommended to be safe in national guidelines. $60 \%$ of attendances to the department were judged to be inappropriate.

Subsequently the ENT emergency service was changed to an appointment based Rapid Access Clinic, with an easily accessible and prompt triage facility provided by a trained triage nurse. Concurrently the opportunity was taken to improve record keeping and formalise post consultation communication.

Re-audit confirmed a 43\% reduction in the number of patients accessing the ENT emergency clinic facility, allowing individual clinic numbers to fall to safe levels. Average patient waiting times fell by $70 \%$ to 22 minutes. The number of referrals judged to be inappropriate was halved.

The transformation of our service has enabled time and resources to be more effectively directed towards a smaller number of patients, whose needs are more urgent.

\section{Problem}

Patients presenting unpredictably with conditions requiring urgent management place a significant burden on ENT departments. Often assessment requires specialised equipment including good quality lighting, microscope and rigid or flexible endoscopy, and is facilitated by both adequately trained medical and nursing staff. Management of problems such as otitis externa, epistaxis and foreign bodies necessitates convenient access to a variety of medications, tools and devices. A large proportion of conditions are not emergent and patients can wait a short period of time before assessment.

A solution to addressing these issues is the open access ENT casualty department. Clinics commonly run at set times on weekdays and all patients, including those without a referral, are seen. This can lead to significant variation in numbers and waiting times, inconveniencing patients. Unless formally triaged, such systems can be open misuse allowing patients with non-urgent conditions to attend.

\section{Background}

The ENT casualty department has been in operation in the Royal Victoria Hospital, Belfast, a busy teaching hospital, for over 20 years. The service was set up to allow convenient access to specialist treatment of urgent conditions for patients in the Greater Belfast area. Patients were able to attend on weekdays having been sent by their GP and local Accident and Emergency departments. Self referrals or "walk-ins" were also assessed.

The consultations were documented on a single, loosely formatted, sheet of paper. The main copy was forwarded to the patient's general practitioner and a carbon copy retained in the medical records department.

The service was very popular with patients who could find it a lot easier than scheduling an appointment with the GP. For the GP outside of the Greater Belfast area it could be seen as a much more convenient way of accessing ENT input than going through referral pathways in their local district general hospitals. It was also significantly quicker than waiting for an outpatient appointment. As pressures on GP practices increased over recent years, there had been a significant increase in numbers of patients attending with unpredictable waiting times. Cases were often felt to be non-urgent or too complex for the time and expertise available. Consequently clinics frequently ran over time causing undue stress on staff and resources.

Over several years previous attempts had been made to streamline the clinic. Sessions were condensed from all day to morning only, in an attempt to improve efficiency. A triage nurse was introduced to redirect patients whose needs would be better served by a 
consultant outpatient appointment. Advice leaflets and teaching were offered to local General Practitioners.

Despite these efforts the service remained overburdened. An audit was commenced to quantify the numbers of patients attending, source of referral, patterns of conditions and overall appropriateness of referrals.

\section{Baseline Measurement}

An audit was designed to provide an overview of attendances at the Royal Victoria Hospital ENT casualty department. Initially a review of number of attendances over the preceding year was carried out from a daily log. Times patients waited in the department were calculated, over an average one week period, from information in the log. Specifically note was made of time between registration and consultation.

Subsequently a detailed audit was performed over a four week period. Carbon copy notes were reviewed at the end of each morning session by a single investigator. The Health and Social Care Trust in which the patient resided was recorded (based on the postcode of the patient's registered address but excluding post operative patients and those referred from other wards) along with the source of the referral. Presenting complaint, duration of symptoms and interventions at the clinic were documented. An overall judgement of appropriateness of attendance was made based on all information available in both the casualty notes and any referral documentation.

Between October 2008 and September 2009 four thousand, five hundred and seventy seven patients attended the ENT casualty department, averaging 382 per month or 19 per day. Over the four week audit during October and November 2009 daily attendances averaged 17 (range 9 - 22). Patients waited an average of 75 minutes to be assessed by a member of medical staff.

Despite being designed and funded as a local service, $38 \%$ of patients were registered at residential addresses within other Health and Social Care Trust catchment areas. $45 \%$ of attendees had no clear referral source or documentation and hence were considered to be self referrers. $28 \%$ originated from General Practitioners, $6 \%$ from Accident and Emergency departments and 3\% from wards other than the ENT department. $15 \%$ of patients had a planned review appointment.

Patients presented with a wide range of ENT conditions. The most common diagnosis was acute otitis externa (29\%). Approximately one quarter of patients had not yet received any treatment in primary care. Other common diagnoses were epistaxis (8\%), nasal injury ( $8 \%)$ and foreign body (ear, nose or throat) $(7 \%)$. The fifth most common presentation was with wax $(6 \%)$, despite a nurse led de-waxing clinic operating routinely within the department. $18 \%$ of patients had symptoms with greater than 4 weeks duration. One third of these had symptoms for greater than 3 months. 251 procedures, including aural microsuction, audiometry, flexible nasopharyngolaryngoscopy and silver nitrate nasal cautery were performed over the audit period.
An overall judgement of appropriateness of attendances was made by a single investigator. All available documentary evidence was reviewed. $60 \%$ of attendances were deemed to be inappropriate for a variety of reasons. $29 \%$ of patients could have attended emergency ENT facilities within their local hospital and $18 \%$ would have been more appropriately managed through the standard outpatient clinic referral system, either as a consequence of nature of complaint or duration of symptoms. $11 \%$ of patients could have received first line management within a primary care setting.

\section{Design}

From our initial investigation it was clear a large number of patients were attending the ENT casualty service. As a result average daily attendances were in excess of ENT UK recommended safe maximum numbers to be seen at a clinic, by nonconsultant/associate specialist grade, of less than or equal to 12(1). There were long waiting times within the department and excessive stresses on nursing and medical staff and on resources.

A telephone survey, regarding emergency outpatient care, within ENT departments in six NHS trusts in cities with similar population size to Belfast was performed. All hospitals operated an urgent referral clinic and not a walk in service. Review of the literature identified documentation of the establishment of an appointment based urgent care clinic in the Heart of England Trust, Birmingham (2).

Audit findings and a proposal to change to an appointed rapid access clinic were presented at a Northern Ireland Regional audit meeting. Those present were surveyed as to their opinion on ENT emergency management. $97 \%$ agreed patients should ideally be managed in the local hospital and $63 \%$ felt the Royal Victoria Hospital ENT casualty should not remain in its current form. Nursing staff within the department were also surveyed and $100 \%$ felt ENT casualty should not continue in its current state. Of a small selection of local GPs ( $n=12$ ) $92 \%$ supported the proposal to change to an appointment based rapid access clinic.

\section{Strategy}

Following restructuring of ENT in the Belfast Health and Social Care Trust, involving the merging of two units, along with closure of the Belfast City Hospital Accident and Emergency department it was feared an already overburdened emergency ENT service could not cope with anticipated extra demands. Permission for proposals to change to an appointment-based service was granted.

A senior nurse was allocated and trained to provide a triage service. Referrals were to be accepted via direct telephone, fax or email, from GPs, A\&E departments and other non-ENT wards on a standardised form. Referrals would be assessed between 9am and $5 \mathrm{pm}$, Monday to Friday. Triage would allow feedback to the referring healthcare professional and recommendation for treatment to be initiated in primary care if necessary, or at times recommend a more suitable referral pathway. Patients requiring assessment would be contacted directly and offered a timed appointment slot. 
The transformed rapid access clinic was to offer 15 minute appointment slots, with a maximum capacity of 14 per day, five days per week.

Consultation details would now be recorded on a standardised pro forma. A copy would be sent to the GP and a copy filed in the patient's medical notes.

GPs, practice managers and Accident and Emergency departments throughout Northern Ireland were informed in writing of the plans to transform the service. Communication included details of the referral pathway, contact details and guidelines as to which conditions would and would not be suitable for assessment at the clinic. Signposts were placed throughout the hospital informing patients of the plans to change from a walk in facility.

On the 1st November 2011 the Rapid Access Clinic was initiated. Post implementation audit was carried out after an initial settling in period.

\section{Results}

Several months after the ENT Rapid Access clinic opened an audit was performed. The same methodology as previously was used, accessing the rapid access clinic logbook followed by patients' notes, including the newly introduced referral documentation. Information including clinic numbers, referral sources, waiting times and procedures were recorded as previously and a judgement of appropriateness was made.

Between January and September 2012 there were 1985 attendances (34 patients could not attend their appointment and 114 did not attend, giving a non attendance rate of $7 \%$ ). Over the nine month period there were nine statutory holidays and 13 clinics were cancelled as there was no available medical cover. Average attendances per clinic were 11 , a $38 \%$ reduction compared with the previous audit period. This brought attendances in line with guidance for safe clinic numbers issued by ENT UK. During a four week audit during September 2012 fifteen clinics had 200 attendees with an average daily attendance rate of 13 patients.

Patients waited on average 22 minutes between their allotted appointment time and assessment by the medical practitioner.

$32 \%$ of patients continued to originate from addresses outside of the Belfast Health and Social Care Trust, compared with 37\% previously. However given the over all decrease in numbers of attendances this represents an actual reduction of $18 \%$. Self referrals are no longer assessed at the clinic leading to a change in referral source. Now $44 \%$ of patients are referred by their GP and $28 \%$ by Accident and Emergency departments, compared with previous figures of 28 and 6 per cent respectively.

Patients continue to present with a wide variety of complaints and diagnoses. Otitis externa, epistaxis and nasal injury remain the three most common problems. The fourth most common presentation is for vocal cord check prior to thyroid surgery, as this can now be easily facilitated within the current booking system.
Wax impaction is now redirected at triage to the more appropriate nurse led microsuction clinic. Unfortunately we do continue to see $6 \%$ of patients with conditions lasting more than three months. With the decreased number of patients attending, and a change in the patterns of conditions encountered, there has been a $49 \%$ reduction in the number of procedures performed (123 in 2012 versus 251 in 2009) leading to less strain on personnel and resources.

Finally in an overall judgement of appropriateness only $31 \%$ of referrals were felt to be inappropriate, as compared to $60 \%$ in the initial audit. 22\% were from outside the Belfast Trust and could have accessed emergency care in their local area. $9 \%$ would have been more appropriately managed through alternative outpatient referral pathways and only one patient could have been managed in the community by the GP.

\section{Lessons and Limitations}

The ENT emergency facility provides an invaluable resource for patients, GPs, Accident and Emergency departments and non-ENT inpatient wards. Although an initial audit had shown it to be overburdened, with excessive numbers of patients attending and waiting prolonged durations for assessment often without having accessed primary care assessment, there was reticence to change a long established service. It was only with perseverance, and in a backdrop of restructuring within the Belfast Health and Social Care Trust, that proposals for change were adopted.

Although we, and other ENT professionals in the region, feel patients requiring urgent care should be managed locally, we do not refuse to offer appointments to those from outside the Belfast Health and Social Care Trust. The number of patients with prolonged symptoms and those who would be more suited to other referral pathways suggests our triage system could be more robust. It is likely however that simply the existence of triage (a forced function) has reduced attendances.

The judgement of appropriateness is a very crude account of the effectiveness of the department. Although a subjective measure we have attempted to reduce intra-observer variability by using the same observer to review all 530 cases in both audit periods.

\section{Conclusion}

Our initiative to modernise a long standing but inefficient ENT emergency care facility has been a success. The new Rapid Access Clinic provides a safe, effective and timely service to our patients. Time and resources can be more effectively directed towards a smaller number of patients whose needs are urgent. Communication with colleagues in primary care has been standardised, improving continuity of care.

\section{References}

1. ENT UK [Internet]. Recommended numbers in ENT clinics in the UK. Available from: https://entuk.org/docs/prof/misc/Recommended_numbers_in 
ENT clinics

2. Mylvaganam S, Patodi R, Campbell JB. The ENT emergency clinic: a prospective audit to improve effectiveness of an established service. J Laryngol Otol. 2009; 123: 229-233.

\section{Declaration of interests}

Nothing to declare

\section{Acknowledgements}

Sister Joan Gordan

This initiative has been awarded 1st prize in the 2012 Belfast Health and Social Care Trust Chairman's Awards (modernisation category) 